\title{
Nonparametric estimation of conditional quantiles
}

\author{
Alexander Kukush * \\ Jan Beirlant ${ }^{\dagger}$ \\ Yuri Goegebeur
}

July 20,2005

\begin{abstract}
Nonextreme regression quantiles are estimated nonparametrically on the basis of local polynomial approximations to the true conditional quantile function. The consistency of the estimator is shown. The asymptotic normality is proven and the asymptotic confidence interval for the regression quantile is constructed.

Keywords and phrases: Local polynomial estimation; quantile regression; consistency; asymptotic normality.
\end{abstract}

*Department of Applied Economics, K.U.Leuven, Naamsestraat 69, B-3000 Leuven. On leave from Kiev National Taras Shevchenko University, Ukraine.

${ }^{\dagger}$ Department of Mathematics, K.U.Leuven, Celestijnenlaan 200B, 3001 Heverlee, Belgium.

${ }^{\ddagger}$ Faculty of Psychology and Educational Science, K.U.Leuven, Tiensestraat 102, 3000 Leuven, Belgium. 


\section{Introduction}

We consider an unspecified regression model with fixed design points and independent correspondent observations $Y_{i}$ of a response variable. Nonextreme conditional quantiles are estimated nonparametrically using a local version of the Koenker and Bassett (1978) regression quantile methodology. This was a part of a two-step procedure for estimating extreme conditional quantiles, which is proposed in Beirlant et al. (2004b). We modify slightly the first step of the procedure in order to obtain the consistent estimator of the nonextreme quantile. We also prove asymptotic normality of the estimator.

A much more general estimation problem is studied in Fan et al. (1994), where $\left(x_{i}, Y_{i}\right)$ are drawn independently from the distribution of $(X, Y)$. The conditional asymptotic normality of the estimator is shown there, which suggests that such a result holds for specific fixed design points, see p. 436 in Fan et al. (1994). In that paper, the conditional expectation of a check function is supposed to be smooth enough, while in the present paper we do not demand this, and the restrictions on our fixed design points are rather weak.

In Section 2 we present the estimator of regression quantiles based on a local polynomial approximation, give conditions and state the main results - consistency and asymptotic normality of the estimator. Technical proofs are deferred to Section 3, and Section 4 concludes.

\section{Estimator and its asymptotic properties}

\section{$2.1 \quad$ The model}

Consider a random variable $Y$ whose distribution depends on a scalar covariate $x$. Let $F_{Y \mid x}$ denote the conditional distribution of $Y$ given $x$, and for a fixed $\theta \in(0,1)$ the associated quantile function equals

$$
Q(\theta, x):=\inf \left\{y: F_{Y \mid x}(y) \geq \theta\right\} .
$$

Suppose a data set $\left(Y_{1}, x_{1}\right), \ldots,\left(Y_{n}, x_{n}\right)$ of independent observations according to $F_{Y \mid x}$ is given, where $x_{1}, \ldots, x_{n}$ are nonrandom design points. Our goal is to estimate $Q\left(\theta, x^{*}\right)$ for a fixed $x^{*}$. We assume no knowledge about $F_{Y \mid x}$ or $Q$ and follow a nonparametric approach.

The next assumption is about the smoothness of the quantile function with respect to $x$. We fix an integer $p \geq 0$, the degree of the polynomial approximation. Hereafter we often skip $\theta$ and write $Q(x)=Q(\theta, x)$.

Condition (i). There exists a neighborhood $\left(x^{*}-\delta, x^{*}+\delta\right)$, such that $Q(x) \in C^{p}\left(x^{*}-\delta, x^{*}+\delta\right)$, moreover the derivative $Q^{(p)}(x)$ satisfies the 
Hölder condition

$$
\left|Q^{(p)}(x)-Q^{(p)}\left(x^{*}\right)\right| \leq \tilde{H}\left|x-x^{*}\right|^{\gamma}, \quad x \in\left(x^{*}-\delta, x^{*}+\delta\right),
$$

where $\tilde{H}$ and $\gamma$ are unknown positive constants, and $\gamma \leq 1$.

Under Condition $(i)$, we can approximate $Q$ locally by a polynomial of degree $p$ centered around $x^{*}$ in the following way

$$
Q(x)=\sum_{j=0}^{p} \beta_{j}\left(x-x^{*}\right)^{j}+r(x), \quad x \in\left(x^{*}-\delta, x^{*}+\delta\right) .
$$

Here $\beta_{j}=\beta_{j}(\theta):=Q^{(j)}\left(x^{*}\right) / j !, j=0, \ldots, p$, in particular $\beta_{0}=Q\left(\theta, x^{*}\right)$, and

$$
|r(x)| \leq H\left|x-x^{*}\right|^{p+\gamma}, \quad H:=\tilde{H} / p !
$$

We fix a sequence $\left\{h_{n}, n \geq 1\right\}$ of positive numbers which converges to 0 . Consider a window of shrinking size $2 h_{n}$ centered at $x^{*}$ in which we apply the expansion (3). Within this window we approximate the coefficients $\beta_{j}$ 's as follows:

$$
\min _{\mathbf{b} \in \Theta_{n}} \sum_{i=1}^{n} I_{\left[x^{*}-h_{n}, x^{*}+h_{n}\right]}\left(x_{i}\right) f_{\theta}\left(Y_{i}-p\left(\mathbf{b}, x_{i}\right)\right) .
$$

Here $\Theta_{n}$ is an increasing parameter set,

$$
\Theta_{n}:=\left\{\mathbf{b}=\left(b_{0}, \ldots, b_{p}\right): b_{0} \in \mathbb{R},\left|b_{j}\right| \leq L_{j} / h_{n}^{j}, j=1, \ldots, p\right\}
$$

with fixed positive numbers $L_{j}, f_{\theta}$ is a check function, $f_{\theta}(x):=\theta x^{+}+(1-$ $\theta) x^{-}, x^{+}:=\max (0, x), x^{-}:=\max (0,-x)$, and $I_{\mathcal{A}}(x)=1$ if $x \in \mathcal{A}, 0$ otherwise, and $p(\mathrm{~b}, x):=\sum_{j=0}^{p} b_{j}\left(x-x^{*}\right)^{j}$. This optimization problem is a local version of the Koenker and Bassett (1978) approach to estimating regression quantiles, and it is a modification of the one from Beirlant et al. (2004b), where the size of the window does not vary with the sample size and the parameter set in the optimization problem is the total space $\mathbb{R}^{p+1}$.

We mention that the minimum in (5) is always attained since the objective function converges to infinity uniformly in $\left|b_{j}\right| \leq L_{j} / h_{n}^{j}, j=1, \ldots, p$, as $\left|b_{0}\right| \rightarrow \infty$. The estimator of $\boldsymbol{\beta}:=\left(\beta_{0}, \ldots, \beta_{p}\right)$ is a measurable solution $\hat{\boldsymbol{\beta}}:=\left(\hat{\beta}_{0}, \ldots, \hat{\beta}_{p}\right)$ of $(5)$, which always exists.

\subsection{Consistency}

Next we give the conditions for the consistency of $\hat{\beta}_{0}$. Denote by $f_{Y \mid x}$ the conditional density function of $Y$ given $x$. 
Condition (ii). There exist positive $C_{1}, C_{2}$ and $\delta$, such that for all $x \in$ $\left(x^{*}-\delta, x^{*}+\delta\right)$ and all $y \in\left(Q\left(x^{*}\right)-\delta, Q\left(x^{*}\right)+\delta\right)$, the following holds

$$
C_{1} \leq f_{Y \mid x}(y) \leq C_{2}
$$

This condition states that for $x$ near the target point and for $y$ near the quantile $Q\left(\theta, x^{*}\right)$, the conditional density $f_{Y \mid x}(y)$ is away from 0 and infinity. We need also a tail assumption.

Condition (iii). There exist positive $\psi$ and $\delta$, such that for all $x \in\left(x^{*}-\right.$ $\delta, x^{*}+\delta$ ) and all $y>0$, the following inequality holds

$$
P(|Y| \geq y \mid x) \leq \frac{\text { const }}{y^{p+1+\psi}}
$$

In particular, for a fixed $r \in(0, p+1+\psi)$, the conditional moments $\mathrm{E}\left(|Y|^{r} \mid x\right)$ are uniformly bounded with respect to $x \in\left(x^{*}-\delta, x^{*}+\delta\right)$. Denote

$$
I_{n}:=\left\{i=1,2, \ldots, n: x_{i} \in\left[x^{*}-h_{n}, x^{*}+h_{n}\right]\right\} .
$$

We rewrite (5) as

$$
\begin{gathered}
\min _{\mathbf{b} \in \Theta_{n}} H_{n}(\mathbf{b}), \\
H_{n}(\mathbf{b}):=\sum_{i \in I_{n}} f_{\theta}\left(Y_{i}-p(\mathbf{b}, x)\right) .
\end{gathered}
$$

Denote by $n_{h}$ the number of points in $I_{n}$.

Condition (iv). $n_{h} h_{n}^{2 p} \rightarrow \infty$ if $p \geq 1$, and $n_{h} \rightarrow \infty$ if $p=0$.

This means that we have enough points in a neighborhood of $x^{*}$. We need the next assumption only in the case $p \geq 1$. We divide the interval $\left[x^{*}-\right.$ $\left.h_{n}, x^{*}+h_{n}\right]$ into $p+2$ equal parts $I_{k_{n}}:=\left\{i=1, \ldots, n: x_{i} \in\left[x^{*}-h_{n}+\right.\right.$ $\left.\left.\frac{k}{p+2} 2 h_{n}, x^{*}-h_{n}+\frac{k+1}{p+2} 2 h_{n}\right]\right\}$ and count the number of design points in each part.

Condition $(v)$. For each $k=0,1, \ldots, p+1$,

$$
\liminf _{n \rightarrow \infty} \frac{\sharp I_{k_{n}}}{n_{h}}>0 \text {. }
$$

Now, we state the consistency result.

Theorem 1. Assume the conditions $(i)$ to (iv), and in the case $p \geq 1$ assume additionally $(v)$. Then for each $j=0,1, \ldots, p,\left(\hat{\beta}_{j}-\beta_{j}\right) h_{n}^{j} \stackrel{\mathcal{P}}{\rightarrow} 0$, as $n \rightarrow \infty$. In particular $\hat{\beta}_{0} \stackrel{\mathcal{P}}{\rightarrow} Q\left(\theta, x^{*}\right)$, as $n \rightarrow \infty$. 


\subsection{Asymptotic normality for $p=0$}

In the case of the local constant approximation, the objective function (8) is a function of a single argument $b_{0}, b_{0} \in \mathbb{R}$. Denote

$$
\left\{Z_{j} \mid j=1, \ldots, n_{h}\right\}:=\left\{Y_{i} \mid i \in I_{n}\right\},
$$

and consider the order statistics

$$
Z_{1, n_{h}} \leq Z_{2, n_{h}} \leq \ldots \leq Z_{n_{h}, n_{h}} .
$$

From inequalities $H_{n,+}^{\prime}\left(\hat{\beta}_{0}\right) \geq 0$ and $H_{n,-}^{\prime}\left(\hat{\beta}_{0}\right) \leq 0$, where $H_{n,+}^{\prime}$ and $H_{n,-}^{\prime}$ are the right and left derivatives respectively, it is easy to get the next explicit formula for $\hat{\beta}_{0}$ : it is measurable and

$$
\hat{\beta}_{0}=\left\{\begin{array}{l}
Z_{\left[\theta n_{h}\right]+1, n_{h}}, \text { if } \theta n_{h} \notin \mathbb{N} \\
\text { any point from }\left[Z_{\theta n_{h}, n_{h}}, Z_{\theta n_{h}+1, n_{h}}\right], \text { if } \theta n_{h} \in \mathbb{N} .
\end{array}\right.
$$

For instance one can set $\hat{\beta}_{0}=Z_{\left[\theta n_{h}\right]+1, n_{h}}$, provided $n_{h} \geq(1-\theta)^{-1}$.

We introduce further assumptions for the asymptotic normality. First we strengthen 2 .

Condition $(i i)^{*} \cdot f_{Y \mid x^{*}}\left(Q\left(x^{*}\right)\right)>0$, and there exists a positive $\delta$, such that the conditional density $f_{Y \mid x}(y)$ is continuous in $y$ and $x$ on a set

$$
\left\{(y, x): x \in\left(x^{*}-\delta, x^{*}+\delta\right), y \in\left(Q\left(x^{*}\right)-\delta, Q\left(x^{*}\right)+\delta\right)\right\} .
$$

Also, we need a stronger condition than 4 . Remember that $\gamma$ is the exponent in 2 .

Condition $(i v)^{*} . n_{h} \rightarrow \infty$ and $n_{h} h^{2 \gamma} \rightarrow 0$, as $n \rightarrow \infty$.

Theorem 2. Assume that $p=0$ and the conditions $(i),(i i)^{*},(i i i)$ and $(i v)^{*}$ hold. Then $\hat{\beta}_{0}$ satisfies (9), and

$$
\sqrt{n_{h}}\left(\hat{\beta}_{0}-Q\left(x^{*}\right)\right) \stackrel{\mathcal{D}}{\rightarrow} N\left(0, \frac{\theta(1-\theta)}{f_{Y \mid x^{*}}^{2}\left(Q\left(x^{*}\right)\right)}\right) \text {, as } n \rightarrow \infty .
$$

Based on (10), we can construct an asymptotic confidence interval for $Q\left(x^{*}\right)$. Let $1-\alpha$ be a confidence probability, $0<\alpha<1$, and $N_{\alpha / 2}$ be the $1-\alpha / 2$ quantile of the standard normal distribution. Then (10) implies that

$$
\lim _{n \rightarrow \infty} P\left(\left|\hat{\beta}_{0}-Q\left(x^{*}\right)\right| \leq \sqrt{\frac{\theta(1-\theta)}{n_{h}}} \frac{N_{\alpha / 2}}{f_{Y \mid x^{*}}\left(Q\left(x^{*}\right)\right)}\right)=1-\alpha .
$$

The density in the denominator of (11) is unknown. In order to bound it, we need the next additional assumption. 
Condition (vi). There exists a positive $\delta$, such that the conditional density $f_{Y \mid x^{*}}(y)$ is monotonic in $y \in\left(Q\left(x^{*}\right)-\delta, Q\left(x^{*}\right)+\delta\right)$.

Now, from $(i i)^{*}$ we have for certain $\delta_{1}>0$ that

$$
\frac{\partial Q\left(\tilde{\theta}, x^{*}\right)}{\partial \theta}=\frac{1}{f_{Y \mid x^{*}}\left(Q\left(\tilde{\theta}, x^{*}\right)\right)},|\tilde{\theta}-\theta|<\delta_{1},
$$

and due to $(v i)$, the partial derivative $\partial Q\left(\tilde{\theta}, x^{*}\right) / \partial \theta$ is monotonic in $\tilde{\theta} \in$ $\left(\theta-\delta_{2}, \theta+\delta_{2}\right)$, for certain $\delta_{2} \leq \delta$. Then on this interval the quantile $Q\left(\tilde{\theta}, x^{*}\right)$ is either convex or concave as a function of $\tilde{\theta}$. Thus for $\varepsilon$ small enough,

$$
\frac{\partial Q\left(\theta, x^{*}\right)}{\partial \theta} \leq \max _{+,-} \frac{\left|Q\left(\theta \pm \varepsilon, x^{*}\right)-Q\left(\theta, x^{*}\right)\right|}{\varepsilon} .
$$

Relations (11) to (13) imply that

$$
\begin{gathered}
\liminf _{n \rightarrow \infty} P\left\{\left|\hat{\beta}_{0}-Q\left(\theta, x^{*}\right)\right| \leq \sqrt{\frac{\theta(1-\theta)}{n_{h}}} N_{\alpha / 2} \times\right. \\
\left.\max _{+,-} \frac{\left|Q\left(\theta \pm \varepsilon, x^{*}\right)-Q\left(\theta, x^{*}\right)\right|}{\varepsilon}\right\} \geq 1-\alpha .
\end{gathered}
$$

Let $\hat{\beta}_{0}^{\tau}$ be the estimator of $Q\left(\tau, x^{*}\right)$, constructed for $p=0$. By the consistency result stated in Theorem 1 , we have that

$$
\frac{\hat{\beta}_{0}^{\theta \pm \varepsilon}-\hat{\beta}_{0}^{\theta}}{\varepsilon} \stackrel{\mathcal{P}}{\rightarrow} \frac{Q\left(\theta \pm \varepsilon, x^{*}\right)-Q\left(\theta, x^{*}\right)}{\varepsilon} .
$$

From (14) and (15) we finally obtain that for small enough $\varepsilon$,

$$
\liminf _{n \rightarrow \infty} P\left\{Q\left(\theta, x^{*}\right) \in\left[\hat{\beta}_{0}^{\theta}-\ell_{n \varepsilon}, \hat{\beta}_{0}^{\theta}+\ell_{n \varepsilon}\right]\right\} \geq 1-\alpha
$$

where

$$
\ell_{n \varepsilon}:=\sqrt{\frac{\theta(1-\theta)}{n_{h}}} N_{\alpha / 2} \max _{+,-} \frac{\left|\hat{\beta}_{0}^{\theta \pm \varepsilon}-\hat{\beta}_{0}^{\theta}\right|}{\varepsilon} .
$$

Thus under the conditions of Theorem 2 and the additional condition $(v i)$, we constructed the asymptotic confidence interval $\left[\hat{\beta}_{0}^{\theta}-\ell_{n \varepsilon}, \hat{\beta}_{0}^{\theta}+\ell_{n \varepsilon}\right]$ for $Q\left(\theta, x^{*}\right)$.

\subsection{Asymptotic normality for $p \geq 1$}

For the local polynomial approximation of order $p \geq 1$, we change the parameter set $(6)$. We fix $\tau \in(0,1)$ and denote

$$
\tilde{\Theta}_{n}:=\left\{\mathbf{b}=\left(b_{0}, \ldots, b_{p}\right): b_{0} \in \mathbb{R},\left|b_{j}\right| \leq L_{j} / h_{n}^{j-\tau}, j=1, \ldots, p\right\},
$$

with fixed positive numbers $L_{j}$. Now the estimator $\tilde{\boldsymbol{\beta}}=\left(\tilde{\beta}_{0}, \ldots, \tilde{\beta}_{p}\right)$ comes from the optimization procedure (5), where $\Theta_{n}$ is replaced by $\tilde{\Theta}_{n}$. We need a new condition on $h_{n}$. 
Condition (vii). $\sqrt{n_{h}} h_{n}^{\tau} \rightarrow 0$, and $\sqrt{n_{h}} h_{n}^{\gamma} \rightarrow 0$, as $n \rightarrow \infty$.

This condition holds e.g. if $h_{n}=n^{-\varepsilon}, \varepsilon>(1+2 \min (\tau, \gamma))^{-1}$ and the ratio $n_{h} /\left(n h_{n}\right)$ is separated from 0 and infinity.

Theorem 3. Assume the conditions $(i),(i i)^{*},(i i i)$ and (vii). Then

$$
\sqrt{n_{h}}\left(\tilde{\beta}_{0}-Q\left(x^{*}\right)\right) \stackrel{\mathcal{D}}{\rightarrow} N\left(0, \frac{\theta(1-\theta)}{f_{Y \mid x^{*}}^{2}\left(Q\left(x^{*}\right)\right)}\right) \text {, as } n \rightarrow \infty .
$$

Based on Theorem 3, the asymptotic confidence interval for $Q\left(x^{*}\right)$ can be constructed like in Section 2.3.

\section{Proofs}

Denote $\bar{B}(0, R):=\left\{\mathbf{x} \in \mathbb{R}^{p}:\|\mathbf{x}\| \leq R\right\}, p \geq 1$.

Lemma 1. (Ibragimov and Hasminski, 1981).

Let $T(\boldsymbol{\beta})$ be a separable, measurable stochastic field defined on a closed set $K \subset \mathbb{R}^{p}$. Suppose that for any $\boldsymbol{\beta}, \tilde{\boldsymbol{\beta}}$ (for which $\boldsymbol{\beta} \in K, \boldsymbol{\beta}+\tilde{\boldsymbol{\beta}} \in K$ ),

$$
E|T(\boldsymbol{\beta}+\tilde{\boldsymbol{\beta}})-T(\boldsymbol{\beta})|^{r} \leq L\|\tilde{\boldsymbol{\beta}}\|^{r^{\prime}},
$$

for some $r \geq r^{\prime}>p$ and a constant $L$. Then for any $a>0$ and $R>0$,

$$
P\left\{\sup _{\boldsymbol{\beta}_{1}, \boldsymbol{\beta}_{\mathbf{2}} \in K \cap \bar{B}(0, R)}\left|T\left(\boldsymbol{\beta}_{1}\right)-T\left(\boldsymbol{\beta}_{2}\right)\right|>a\right\} \leq k_{0} L R^{r^{\prime}} a^{-r},
$$

where $k_{0}$ depends on $r, r^{\prime}, p$ but does not depend on $K, L, R$ and $a$.

Lemma 2. Assume the conditions (i) to (iv). Then $\hat{\beta}_{0}=\hat{\beta}_{0}(n)$ is stochastically bounded.

Proof. We divide it into several parts.

1. Expansion for $H_{n}(\mathbf{b})-H_{n}(\boldsymbol{\beta})$.

For real $Y, Z$ and $q, Z>q$, we have an identity

$$
\begin{aligned}
& f_{\theta}(Y-Z)-f_{\theta}(Y-q)= \\
& \quad(Z-Y) I(q \leq Y \leq Z)+(Z-q)[-\theta I(Y \geq q)+(1-\theta) I(Y<q)] .
\end{aligned}
$$

Here $I(A)=1$ if $A$ holds, 0 otherwise. Consider $b_{0} \geq B$, where we will choose $B$ later. Remember that $\Theta_{n}$ is defined in (6). For $\mathbf{b}=\left(b_{0}, \ldots, b_{p}\right) \in \Theta_{n}$ and $i \in I_{n}$ we have

$$
p\left(\mathbf{b}, x_{i}\right)-p\left(\boldsymbol{\beta}, x_{i}\right) \geq B-\beta_{0}-2 \sum_{j=1}^{p} L_{j}>0
$$




$$
B>\beta_{0}+2 \sum_{j=1}^{p} L_{j}
$$

Here we suppose that $n$ is large enough to have $\left|\beta_{j}\right| \leq L_{j} / h_{n}^{j}$, for all $j=$ $1, \ldots, p$. Now we assume that (20) holds and use (18) for $Y=Y_{i}, Z=$ $p\left(\mathbf{b}, x_{i}\right), q=p\left(\boldsymbol{\beta}, x_{i}\right), i \in I_{n}$. Then for the increment of the objective function (8) we have a representation

$$
H_{n}(\mathbf{b})-H_{n}(\boldsymbol{\beta})=S_{1 n}+S_{2 n}+S_{3 n}+S_{4 n},
$$

with

$$
\begin{aligned}
S_{1 n}:= & \sum_{i \in I_{n}} \mathrm{E}\left(p\left(\mathbf{b}, x_{i}\right)-Y_{i}\right) I\left(p\left(\boldsymbol{\beta}, x_{i}\right) \leq Y_{i} \leq p\left(\mathbf{b}, x_{i}\right)\right), \\
S_{2 n}:= & \sum_{i \in I_{n}}\left[\left(p\left(\mathbf{b}, x_{i}\right)-Y_{i}\right) I\left(p\left(\boldsymbol{\beta}, x_{i}\right) \leq Y_{i} \leq p\left(\mathbf{b}, x_{i}\right)\right)-\right. \\
& \left.\mathrm{E}\left(p\left(\mathbf{b}, x_{i}\right)-Y_{i}\right) I\left(p\left(\boldsymbol{\beta}, x_{i}\right) \leq Y_{i} \leq p\left(\mathbf{b}, x_{i}\right)\right)\right], \\
S_{3 n}:= & \sum_{i \in I_{n}}\left(p\left(\mathbf{b}, x_{i}\right)-p\left(\boldsymbol{\beta}, x_{i}\right)\right)\left[-\theta I\left(Y \geq Q\left(x_{i}\right)\right)+\right. \\
& \left.(1-\theta) I\left(Y_{i}>Q\left(x_{i}\right)\right)\right], \\
S_{4 n}:= & \sum_{i \in I_{n}}\left(p\left(\mathbf{b}, x_{i}\right)-p\left(\boldsymbol{\beta}, x_{i}\right)\right)\left[-\theta\left(I\left(Y_{i} \geq p\left(\boldsymbol{\beta}, x_{i}\right)\right)-\right.\right. \\
& \left.I\left(Y_{i} \geq Q\left(x_{i}\right)\right)\right)+(1-\theta)\left(I\left(Y_{i}<p\left(\boldsymbol{\beta}, x_{i}\right)\right)-\right. \\
& \left.\left.I\left(Y_{i}<Q\left(x_{i}\right)\right)\right)\right] .
\end{aligned}
$$

We want to show that with probability tending to $1, \inf _{b_{0} \geq B_{0}, \mathbf{b} \in \Theta_{n}}\left(H_{n}(\mathbf{b})-\right.$ $\left.H_{n}(\boldsymbol{\beta})\right) / n_{h}$ is positive and separated from 0 .

\section{Behavior of $S_{1 n}$}

Let $F_{i}$ be the distribution function $F_{Y \mid x_{i}}$, and $\bar{F}_{i}:=1-F_{i}$ be the tail probability. Then

$$
\mathrm{E}\left(p\left(\mathbf{b}, x_{i}\right)-Y_{i}\right) I_{\left[p\left(\boldsymbol{\beta}, x_{i}\right), p\left(\mathbf{b}, x_{i}\right)\right]}\left(Y_{i}\right)=-\int_{p\left(\boldsymbol{\beta}, x_{i}\right)}^{p\left(\mathbf{b}, x_{i}\right)}\left(p\left(\mathbf{b}, x_{i}\right)-t\right) d \bar{F}_{i}(t) .
$$

Under $(i i)$, we may and do assume that $F_{i}$ is continuous at the point $p\left(\boldsymbol{\beta}, x_{i}\right)$; we can assume the same at the point $p\left(\mathbf{b}, x_{i}\right)$. Then for $i \in I_{n}$,

$$
\begin{aligned}
& \mathrm{E}\left(p\left(\mathbf{b}, x_{i}\right)-Y_{i}\right) I_{\left[p\left(\boldsymbol{\beta}, x_{i}\right), p\left(\mathbf{b}, x_{i}\right)\right]}\left(Y_{i}\right)= \\
& \quad\left(p\left(\mathbf{b}, x_{i}\right)-p\left(\boldsymbol{\beta}, x_{i}\right)\right) \bar{F}_{i}\left(p\left(\boldsymbol{\beta}, x_{i}\right)\right)-\int_{p\left(\boldsymbol{\beta}, x_{i}\right)}^{p\left(\mathbf{b}, x_{i}\right)} \bar{F}_{i}(t) d t .
\end{aligned}
$$


Next, under $(i i), \bar{F}_{i}\left(p\left(\boldsymbol{\beta}, x_{i}\right)\right)=\bar{F}_{i}\left(Q\left(x_{i}\right)\right)+O\left(h_{n}^{\gamma}\right)=1-\theta+O\left(h_{n}^{\gamma}\right)$, where $O\left(h_{n}^{\gamma}\right)$ is uniform with respect to the index $i$. From (iii) it follows that the integral on the right hand side of (23) is uniformly bounded. Then (23) equals $\left(p\left(\mathbf{b}, x_{i}\right)-p\left(\boldsymbol{\beta}, x_{i}\right)\right)(1-\theta)+o\left(b_{0}\right)$, as $b_{0} \rightarrow+\infty$, where $o\left(b_{0}\right)$ is uniform in $i$. Then

$$
S_{1 n}=(1-\theta) \sum_{i \in I_{n}}\left(p\left(\mathbf{b}, x_{i}\right)-p\left(\boldsymbol{\beta}, x_{i}\right)\right)+n_{h} o\left(b_{0}\right),
$$

and

$$
S_{1 n}=(1-\theta) b_{0} n_{h}+o(1) n_{h} b_{0}, \text { as } b_{0} \rightarrow+\infty .
$$

\section{Behavior of $S_{2 n}$}

We assume that positive $B$ satisfies (20) and for $b_{0} \geq B_{0}, \mathbf{b} \in \Theta_{n}$, denote $\alpha_{0}:=1 / b_{0}, \alpha_{j}:=b_{j} / b_{0}, j=1, \ldots, p$. Then

$$
\left(\alpha_{0}, \ldots, \alpha_{p}\right) \in \Theta_{0}:=\left[0, \frac{1}{B_{0}}\right] \times \prod_{j=1}^{p}\left[-\frac{L_{j}}{B_{0} h_{n}^{j}}, \frac{L_{j}}{B_{0} h_{n}^{j}}\right] .
$$

Denote

$$
\begin{aligned}
Z(\boldsymbol{\alpha}):= & \frac{1}{b_{0}} S_{2 n}=\sum_{i \in I_{n}}\left(\xi_{i}(\boldsymbol{\alpha})-\mathrm{E} \xi_{i}(\boldsymbol{\alpha})\right), \boldsymbol{\alpha} \in \Theta_{0}, \alpha_{0}>0, \\
\xi_{i}(\boldsymbol{\alpha}):= & \left(1+\sum_{j=1}^{p} \alpha_{j}\left(x_{i}-x^{*}\right)^{j}-\alpha_{0} Y_{i}\right) I\left(p\left(\boldsymbol{\beta}, x_{i}\right) \leq Y_{i} \leq\right. \\
& \left.\frac{1}{\alpha_{0}}+\sum_{j=1}^{p} \frac{\alpha_{j}}{\alpha_{0}}\left(x_{i}-x^{*}\right)^{j}\right) .
\end{aligned}
$$

At point $\alpha_{0}=0$ we define

$$
\begin{aligned}
Z\left(0, \alpha_{1}, \ldots, \alpha_{p}\right) & :=\sum_{i \in I_{n}}\left(\xi_{i}\left(0, \alpha_{1}, \ldots, \alpha_{p}\right)-\mathrm{E} \xi_{i}\left(0, \alpha_{1}, \ldots, \alpha_{p}\right)\right), \\
\xi_{i}\left(0, \alpha_{1}, \ldots, \alpha_{p}\right) & :=\left(1+\sum_{j=1}^{p} \alpha_{j}\left(x_{i}-x^{*}\right)^{j}\right) I\left(p\left(\boldsymbol{\beta}, x_{i}\right) \leq Y_{i}\right) .
\end{aligned}
$$

We want to bound $Z(\boldsymbol{\alpha}), \alpha \in \Theta_{0}$, based on Lemma 1 and have to check the moment condition (16). The field $Z(\boldsymbol{\alpha})$ is the most sensible with respect to $\alpha_{0}$, therefore we consider the increments of $Z(\alpha)$ only for varying $\alpha_{0}$. Let $\alpha_{0}>0$ and consider

$$
\begin{aligned}
& Z(\boldsymbol{\alpha})-Z\left(0, \alpha_{1}, \ldots, \alpha_{p}\right)= \\
& \sum_{i \in I_{n}}\left[\left(\xi_{i}(\boldsymbol{\alpha})-\xi_{i}\left(0, \alpha_{1}, \ldots, \alpha_{p}\right)\right)-\mathrm{E}\left(\xi(\boldsymbol{\alpha})-\xi_{i}\left(0, \alpha_{1}, \ldots, \alpha_{p}\right)\right)\right],
\end{aligned}
$$




$$
\begin{aligned}
& \xi_{i}(\boldsymbol{\alpha})-\xi_{i}\left(0, \alpha_{1}, \ldots, \alpha_{p}\right)= \\
& \quad-\left(1+\sum_{j=1}^{p} \alpha_{j}\left(x_{i}-x^{*}\right)^{j}\right) I\left(Y_{i}>\frac{1}{\alpha_{0}}+\sum_{j=1}^{p} \frac{\alpha_{j}}{\alpha_{0}}\left(x_{i}-x^{*}\right)^{j}\right) \\
& -\alpha_{0} Y_{i} I\left(p\left(\boldsymbol{\beta}, x_{i}\right) \leq Y_{i} \leq \frac{1}{\alpha_{0}}+\sum_{j=1}^{p} \frac{\alpha_{j}}{\alpha_{0}}\left(x_{i}-x^{*}\right)^{j}\right)=:-\eta_{i 1}-\eta_{i 2} .
\end{aligned}
$$

We use the Rosenthal inequality for the exponent $r \geq 2, r>p+1$, see Rosenthal (1970). We have

$$
\mathrm{E}\left|Z(\boldsymbol{\alpha})-Z\left(0, \alpha_{1}, \ldots, \alpha_{p}\right)\right|^{r} \leq \mathrm{const} n_{h}^{r / 2} \sup _{i \in I_{n}} \mathrm{E}\left|\eta_{i 1}+\eta_{i 2}\right|^{r} .
$$

Now, $\left\{\eta_{i 1}\right\}$ are uniformly bounded, and

$$
\mathrm{E}\left|\eta_{i 2}\right|^{r}=\alpha_{0}^{r} \mathrm{E}\left|Y_{i}\right|^{r} I\left(p\left(\boldsymbol{\beta}, x_{i}\right) \leq Y_{i} \leq \frac{1}{\alpha_{0}}+\frac{1}{B_{0} \alpha_{0}} O(1)\right) .
$$

Due to (iii) we have for $u \geq p\left(\boldsymbol{\beta}, x_{i}\right), u>0$ :

$$
\mathrm{E}\left|Y_{i}\right|^{r} I\left(p\left(\boldsymbol{\beta}, x_{i}\right) \leq Y_{i} \leq u\right) \leq \mathrm{const}\left(1+u^{r-p-\psi-1}\right)
$$

Then for $r \geq 2, r \geq p+\psi+1$,

$$
\mathrm{E}\left|\eta_{i 2}\right|^{r} \leq \text { const }\left(\alpha_{0}^{p+\psi+1}+\alpha_{0}^{r}\right) .
$$

Thus from (25), (26) for $\boldsymbol{\alpha} \in \Theta_{0}, \boldsymbol{\alpha}^{\prime}=\left(0, \alpha_{1}, \ldots, \alpha_{p}\right) \in \Theta_{0}$ we have

$$
\mathrm{E}\left|Z(\boldsymbol{\alpha})-Z\left(\boldsymbol{\alpha}^{\prime}\right)\right|^{r} \leq \mathrm{const} n_{h}^{r / 2}\left\|\boldsymbol{\alpha}-\boldsymbol{\alpha}^{\prime}\right\|^{p+\psi+1} .
$$

It is not difficult to check that (27) holds as well for any $\boldsymbol{\alpha} \in \Theta_{0}, \boldsymbol{\alpha}^{\prime}=$ $\left(\alpha_{0}^{\prime}, \alpha_{1}, \ldots, \alpha_{p}\right) \in \Theta_{0}$, and we can propagate (27) for any points $\boldsymbol{\alpha}, \boldsymbol{\alpha}^{\prime} \in \Theta_{0}$. By Lemma 1 , for any $a>0$ we have

$$
P\left\{\sup _{\boldsymbol{\alpha}^{\prime}, \boldsymbol{\alpha}^{\prime \prime} \in \Theta_{0}}\left|Z\left(\boldsymbol{\alpha}^{\prime}\right)-Z\left(\boldsymbol{\alpha}^{\prime \prime}\right)\right|>a\right\} \leq \text { const } n_{h}^{r / 2} a^{-r} R^{p+\psi+1},
$$

with

$$
R=\max _{\mathbf{b} \in \Theta_{0}}\|\mathbf{b}\| \leq \text { const } h_{n}^{-p}
$$

Now,

$$
Z(0)=\sum_{i \in I_{n}}\left[I\left(Y_{i} \geq p\left(\boldsymbol{\beta}, x_{i}\right)\right)-P\left(Y_{i} \geq p\left(\boldsymbol{\beta}, x_{i}\right)\right)\right]=\sqrt{n_{h}} O_{p}(1)
$$


Hereafter $O_{p}(1)$ denotes a sequence of stochastically bounded random variables, and this boundedness is uniform with respect to relevant parameters. Then (28) to (30) imply that

$$
\sup _{\boldsymbol{\alpha} \in \Theta_{0}}|Z(\boldsymbol{\alpha})|=\sqrt{n_{h}} \frac{O_{p}(1)}{h_{n}^{(p+\psi+1) p / r}} .
$$

Since $r$ can be chosen large enough, for any fixed $\mu>0$ we obtain

$$
\sup _{\boldsymbol{\alpha} \in \Theta_{0}}|Z(\boldsymbol{\alpha})|=\sqrt{n_{h}} O_{p}(1) h_{n}^{-\mu I(p \geq 1)} .
$$

Thus

$$
S_{2 n}=b_{0} \sqrt{n_{h}} O_{p}(1) h_{n}^{-\mu I(p \geq 1)} .
$$

\section{Behavior of $S_{3 n}$ and $S_{4 n}$}

Denote $\varepsilon_{i}:=-\theta I\left(Y_{i} \geq Q\left(x_{i}\right)\right)+(1-\theta) I\left(Y_{i}<Q\left(x_{i}\right)\right), i \in I_{n}$. Due to $(i i)$ the distribution function of $Y_{i}$ is continuous at the point $Q\left(x_{i}\right)$, therefore $\mathrm{E} \varepsilon_{i}=0$, and

$$
S_{3 n}=\sum_{j=0}^{p}\left(b_{j}-\beta_{j}\right) \sum_{i \in I_{n}}\left(x_{i}-x^{*}\right)^{j} \varepsilon_{i} .
$$

Bounding the second moment of the sum we get

$$
\sum_{i \in I_{n}}\left(x_{i}-x^{*}\right)^{j} \varepsilon_{i}=\sqrt{n_{h}} h_{n}^{j} O_{p}(1) .
$$

Remember that $\left|b_{j}-\beta_{j}\right| \leq 2 L_{j} h_{n}^{-j}, j=1, \ldots, p$. Therefore

$$
S_{3 n}=\left(b_{0}-\beta_{0}\right) \sqrt{n_{h}} O_{p}(1)+\sqrt{n_{h}} O_{p}(1) .
$$

Next, we have

$$
\left|S_{4 n}\right| \leq\left(b_{0}+\text { const }\right) \sum_{i \in I_{n}} I\left(Y_{i} \text { lies between } p\left(\boldsymbol{\beta}, x_{i}\right) \text { and } Q\left(x_{i}\right)\right) .
$$

But by the conditions $(i)$ and $(i i)$,

$$
\mathrm{E} I\left(Y_{i} \text { lies between } p\left(\boldsymbol{\beta}, x_{i}\right) \text { and } Q\left(x_{i}\right)\right) \leq \text { const } h_{n}^{\gamma} .
$$

Then

$$
\left|S_{4 n}\right| \leq\left(b_{0}+\text { const }\right) n_{h} h_{n}^{\gamma} O_{p}(1) .
$$

\section{End of the proof}


Summarizing (21), (24), (31), (32) and (33), we obtain for large enough $B$ that

$\inf _{b_{0} \geq B, \mathbf{b} \in \Theta_{n}} \frac{H_{n}(\mathbf{b})-H_{n}(\boldsymbol{\beta})}{n_{h}} \geq B\left(\frac{1-\theta}{2}+o_{p}(1)+\frac{O_{p}(1)}{\sqrt{n_{h}} h_{n}^{\mu I(p \geq 1)}}\right)+o_{p}(1),(34$

where $o_{p}(1)$ and $O_{p}(1)$ are uniform with respect to $B$. By condition (iv), $\sqrt{n_{h}} h_{n}^{\mu I(p \geq 1)} \rightarrow \infty$ for suitably chosen $\mu>0$. Then from (34) for large enough $B$ we have

$$
\lim _{n \rightarrow \infty} P\left(\inf _{b_{0} \geq B, \mathbf{b} \in \Theta_{n}}\left(H_{n}(\mathbf{b})-H_{n}(\boldsymbol{\beta})\right)>0\right)=1 .
$$

Therefore $P\left(\hat{\beta}_{0}(n) \geq B\right) \rightarrow 0$, as $n \rightarrow \infty$. Similarly for large enough $\bar{B}$, $P\left(\hat{\beta}_{0}(n) \leq-\bar{B}\right) \rightarrow 0$, as $n \rightarrow \infty$. Thus

$$
\lim _{n \rightarrow \infty} P\left(\hat{\beta}_{0}(n) \in[-\bar{B}, B]\right)=1,
$$

which proves Lemma 2 .

Lemma 3. (Contrast inequality) Let $Y$ be a random variable, $\theta \in(0,1)$, and $Q:=\inf \left\{y: F_{Y}(y) \geq \theta\right\}$ be the $\theta$ quantile. Suppose that there exists a positive $\delta$, such that $Y$ has a probability density $f_{Y}(t)$ for $|t-Q|<\delta$, and

$$
c:=\inf _{|t-Q|<\delta} f_{Y}(t)>0 .
$$

Then for each $Z \in \mathbb{R}$,

$$
E\left(f_{\theta}(Y-Z)-f_{\theta}(Y-Q)\right) \geq \frac{c}{2} \min \left(\delta^{2},(Z-Q)^{2}\right) .
$$

Proof. Due to the assumption, $F_{Y}$ is continuous at point $y=Q$. Then from the definitions of $f_{\theta}$ and $Q$ we have

$\varepsilon_{Z}:=\mathrm{E}\left(f_{\theta}(Y-Z)-f_{\theta}(Y-Q)\right)=\mathrm{E}|Y-Z| I(Y$ lies between $Z$ and $Q) .(36)$

Here in case $Z>Q$ we have $I(Q<Y \leq Z)$, and in case $Z<Q$ we have $I(Z \leq Y<Q)$. In the first case, for $Q<Z \leq Q+\delta$

$$
\varepsilon_{Z}=\int_{Q}^{Z}(Z-t) f_{Y}(t) d t \geq \frac{c}{2}(Z-Q)^{2} .
$$

From (36) it follows that $\varepsilon_{Z}$ is increasing in $Z \in[Q,+\infty)$. Therefore for any $Z \geq Q, \varepsilon_{Z} \geq \frac{c}{2} \min \left(\delta^{2},(Z-Q)^{2}\right)$. The case $Z<Q$ is treated similarly.

Fix $k \in \mathbb{N}$. Denote by $\mathcal{P}_{k}$ the space of all real polynomials of degree at most $k ; \mathcal{P}_{k}[a, b]$ is the space of all such polynomials on $[a, b]$. 
Lemma 4. There exists a positive $\delta_{k}$, such that for each $p(x)=a_{0}+a_{1} x+$ $\cdots+a_{k} x^{k} \in \mathcal{P}_{k}$ with $a_{0}^{2}+a_{1}^{2}+\cdots+a_{k}^{2} \geq 1$ there exists $m=0,1, \ldots, k+1$, for which

$$
\min _{\frac{m}{k+2} \leq x \leq \frac{m+1}{k+2}}|p(x)| \geq \delta_{k} .
$$

Proof. Consider an arbitrary interval $\left[\frac{m}{k+2}, \frac{m+1}{k+2}\right], m=0,1, \ldots, k+1$. On the space $\mathcal{P}_{k}\left[\frac{m}{k+2}, \frac{m+1}{k+2}\right]$ the next two norms are equivalent

$$
\|p\|_{\infty, m}:=\max _{\frac{m}{k+2} \leq x \leq \frac{m+1}{k+2}}|p(x)|, \text { and }\|p\|_{2}:=\sqrt{\sum_{j=0}^{k} a_{j}^{2}}
$$

Here $p(x)=\sum_{j=0}^{k} a_{j} x^{j}$. Hence there exists $L_{k m}>0$, such that $\|p\|_{2} \leq$ $L_{k m}\|p\|_{\infty, m}$ for each $p \in \mathcal{P}_{k}$. Let $L_{k}:=\max _{0 \leq m \leq k+1} L_{k m}$ and $\delta_{k}:=1 / L_{k}$. Consider arbitrary $p(x)=a_{0}+\cdots+a_{k} x^{k} \in \mathcal{P}_{k}$ with $a_{0}^{2}+\cdots+a_{k}^{2} \geq 1$. Then $\|p\|_{2} \geq 1$, and for each $m,\|p\|_{\infty, m} \geq \delta_{k}$. Therefore there exist the points $\xi_{m} \in\left[\frac{m}{k+2}, \frac{m+1}{k+2}\right]$, such that $\left|p\left(\xi_{m}\right)\right| \geq \delta_{k}, m=0,1, \ldots, k+1$. We may and do assume that $\xi_{m} \in\left(\frac{m}{k+2}, \frac{m+1}{k+2}\right), 0 \leq m \leq k+1$ (for that purpose we could divide $[0,1]$ into smaller equal parts and select smaller $\delta_{k}$ ). Now suppose that for each $m, \min _{\left[\frac{m}{k+2}, \frac{m+1}{k+2}\right]}|p(x)|<\delta_{k}$, then for each $m$ there exists $\eta_{m} \in\left[\frac{m}{k+2}, \frac{m+1}{k+2}\right]$ with $\left|p\left(\eta_{m}\right)\right|=\delta_{k}$. The properties of $\left\{\xi_{m}\right\}$ and $\left\{\eta_{m}\right\}$ imply that $p(x)$ has at least $k+1$ intervals of strict monotonicity on $[0,1]$, which is impossible for a polynomial of degree at most $k$. The contradiction completes the proof.

Proof of Theorem 1 We divide the proof into several parts.

\section{Proof. 1. Asymptotically equivalent optimization problem}

Due to relation (35), we can restrict the parameter set (6) to

$$
\Theta_{n}^{1}:=\left\{\mathbf{b} \in \mathbb{R}^{p+1}:\left|b_{j}\right| \leq L_{j} / h_{n}^{j}, j=0, \ldots, p\right\},
$$

where $L_{0}:=\max \left(\bar{B}, B,\left|\beta_{0}\right|\right), \bar{B}$ and $B$ come from (35). We do not change the objective function $H_{n}(\mathbf{b})$ given in $(8)$. The estimator $\hat{\boldsymbol{\beta}}(n)$ was defined by (7), (8), and with probability tending to $1, \hat{\boldsymbol{\beta}}(n)=\overline{\boldsymbol{\beta}}(n):=$ $\arg \min _{\mathbf{b} \in \Theta_{n}^{1}} H_{n}(\mathbf{b})$. Thus it is enough to prove that

$$
\left(\bar{\beta}_{j}-\beta_{j}\right) h_{n}^{j} \stackrel{\mathcal{P}}{\rightarrow} 0, \text { as } n \rightarrow \infty ; j=0, \ldots, p .
$$

Here $\left(\bar{\beta}_{0}, \ldots, \bar{\beta}_{p}\right)=\overline{\boldsymbol{\beta}}(n)$. Below we suppress $n$ and write $\overline{\boldsymbol{\beta}}(n)=\overline{\boldsymbol{\beta}}$. 
2. The bound for $p\left(\overline{\boldsymbol{\beta}}, x_{i}\right)-p\left(\boldsymbol{\beta}, x_{i}\right)$

Denote $\Delta_{n}(\mathbf{b}):=\mathrm{E}\left[H_{n}(\mathbf{b})-H_{n}(\boldsymbol{\beta})\right], \mathbf{b} \in \Theta$. Since $\left|\beta_{0}\right| \leq L_{0}$ and $L_{j} / h_{n}^{j} \rightarrow 0$, as $n \rightarrow \infty$, for $j=1, \ldots, p$, we have $\boldsymbol{\beta} \in \Theta_{n}^{1}$, for all $n \geq n_{0}$. Let $n \geq n_{0}$. Then $H_{n}(\overline{\boldsymbol{\beta}}) \leq H_{n}(\boldsymbol{\beta})$, hence

$$
\Delta_{n}(\overline{\boldsymbol{\beta}}) \leq \sup _{\mathbf{b} \in \Theta_{n}^{1}}\left|H_{n}(\mathbf{b})-H_{n}(\boldsymbol{\beta})-\Delta_{n}(\mathbf{b})\right|=: r_{1} .
$$

Now, for each $\mathbf{b} \in \Theta_{n}^{1}$,

$$
\begin{aligned}
\Delta_{n}(\mathbf{b}) \geq & \sum_{i \in I_{n}} \mathrm{E}\left[f_{\theta}\left(Y_{i}-p\left(\mathbf{b}, x_{i}\right)\right)-f_{\theta}\left(Y_{i}-Q\left(\theta, x_{i}\right)\right)\right]-r_{2}, \\
r_{2} & :=\sum_{i \in I_{n}} \mathrm{E}\left|f_{\theta}\left(Y_{i}-Q\left(\theta, x_{i}\right)\right)-f_{\theta}\left(Y_{i}-p\left(\boldsymbol{\beta}, x_{i}\right)\right)\right| \\
& \leq n_{h} \sup _{\left|x-x^{*}\right| \leq h_{n}}|Q(\theta, x)-p(\boldsymbol{\beta}, x)| .
\end{aligned}
$$

From (4) we have

$$
r_{2} \leq \text { const } h_{n}^{p+\gamma} n_{h}
$$

From (39) to (41) we obtain

$$
\left.\sum_{i \in I_{n}} \mathrm{E}\left[f_{\theta}\left(Y_{i}-p\left(\mathbf{b}, x_{i}\right)\right)-f_{\theta}\left(Y_{i}-Q\left(\theta, x_{i}\right)\right)\right]\right|_{\mathbf{b}=\overline{\boldsymbol{\beta}}} \leq r_{1}+\text { const } h_{n}^{p+\gamma} n_{h}
$$

Then from condition (ii) and Lemma 3 we get

$$
\sum_{i \in I_{n}}\left(p\left(\overline{\boldsymbol{\beta}}, x_{i}\right)-Q\left(\theta, x_{i}\right)\right)^{2} \leq \mathrm{const} r_{1}+\text { const } h_{n}^{p+\gamma} n_{h} .
$$

By (4), this implies that

$$
\sum_{i \in I_{n}}\left(p\left(\overline{\boldsymbol{\beta}}, x_{i}\right)-p\left(\boldsymbol{\beta}, x_{i}\right)\right)^{2} \leq \text { const } r_{1}+\text { const } h_{n}^{p+\gamma} n_{h} .
$$

Next, we bound the residual $r_{1}$. Let

$$
T_{n}(\mathbf{b}):=H_{n}(\mathbf{b})-\mathrm{E}\left(H_{n}(\mathbf{b})\right), \mathbf{b} \in \Theta_{n}^{1} .
$$

Fix $r>p+1, r \geq 2$. Since $T_{n}(\mathbf{b})$ is a sum of independent random variables with zero expectation, we can apply the Rosenthal moment inequality, see Rosenthal (1970). We have for $\mathbf{b}_{1}, \mathbf{b}_{2} \in \Theta_{n}^{1}$ that

$$
\mathrm{E}\left|T_{n}\left(\mathbf{b}_{1}\right)-T_{n}\left(\mathbf{b}_{2}\right)\right|^{r} \leq \mathrm{const} n_{h}^{r / 2} \Lambda_{n}\left(\mathbf{b}_{1}, \mathbf{b}_{2}\right)
$$


with

$$
\begin{aligned}
\Lambda_{n}\left(\mathbf{b}_{1}, \mathbf{b}_{2}\right):= & \max _{i \in I_{n}} \mathrm{E} \mid f_{\theta}\left(Y_{i}-p\left(\mathbf{b}_{1}, x_{i}\right)\right)-f_{\theta}\left(Y_{i}-p\left(\mathbf{b}_{2}, x_{i}\right)\right)- \\
& \operatorname{E} f_{\theta}\left(Y_{i}-p\left(\mathbf{b}_{\mathbf{1}}, x_{i}\right)\right)+\left.\operatorname{E} f_{\theta}\left(Y_{i}-p\left(\mathbf{b}_{2}, x_{i}\right)\right)\right|^{r} .
\end{aligned}
$$

Next, $\left|p\left(\mathbf{b}_{1}, x_{i}\right)-p\left(\mathbf{b}_{2}, x_{i}\right)\right| \leq$ const $\left\|\mathbf{b}_{1}-\mathbf{b}_{2}\right\|, i \in I_{n}$. Then (45) and (46) imply that

$$
\mathrm{E}\left|T_{n}\left(\mathbf{b}_{1}\right)-T_{n}\left(\mathbf{b}_{2}\right)\right|^{r} \leq \mathrm{const} n_{h}^{r / 2}\left\|\mathbf{b}_{1}-\mathbf{b}_{2}\right\| .
$$

We apply Lemma 1 with $r^{\prime}=r, L=$ const $n_{h}^{r / 2}$ and

$$
R=\max _{\mathbf{b} \in \Theta_{n}^{1}}\|\mathbf{b}\| \leq \frac{\text { const }}{h_{n}^{p}} .
$$

We have for any $a>0$,

$$
P\left\{\sup _{\mathbf{b}_{1}, \mathbf{b}_{2} \in \Theta_{n}^{1}}\left|T_{n}\left(\mathbf{b}_{1}\right)-T_{n}\left(\mathbf{b}_{2}\right)\right|>a\right\} \leq \text { const } n_{h}^{r / 2} h_{n}^{-r p} a^{-r} .
$$

Let $b=a h_{n}^{p} / \sqrt{n_{h}}$. Then

$$
P\left\{\frac{h_{n}^{p}}{\sqrt{n_{h}}} \sup _{\mathbf{b}_{1}, \mathbf{b}_{2} \in \Theta_{n}^{1}}\left|T_{n}\left(\mathbf{b}_{1}\right)-T_{n}\left(\mathbf{b}_{2}\right)\right|>b\right\} \leq \frac{\text { const }}{b^{r}} .
$$

Thus, see (39),

$$
r_{1}=\frac{\sqrt{n_{h}}}{h_{n}^{p}} O_{p}(1)
$$

and due to (43) and condition (iv),

$$
\begin{aligned}
\varepsilon_{n} & :=\sum_{i \in I_{n}}\left(p\left(\overline{\boldsymbol{\beta}}, x_{i}\right)-p\left(\boldsymbol{\beta}, x_{i}\right)\right)^{2} \leq \frac{\sqrt{n_{h}}}{h_{n}^{p}} O_{p}(1)+\text { const } h_{n}^{p+\gamma} n_{h} \\
& =n_{h} o_{p}(1) .
\end{aligned}
$$

\section{Consistency}

Rescale the parameters and introduce $d_{j}:=\beta_{j} h_{n}^{j}, j=0, \ldots, p ; t_{i}:=\frac{x_{i}-x^{*}}{2 h_{n}}+$ $\frac{1}{2}, i \in I_{n} ; q(\mathbf{d}, t):=d_{0}+d_{1} t+\cdots+d_{p} t^{p}, \mathbf{d}:=\left(d_{0}, \ldots, d_{p}\right)$. Also $\bar{d}_{j}:=\bar{\beta}_{j} h_{n}^{j}$, $j=0, \ldots, p ; \overline{\mathbf{d}}:=\left(\bar{d}_{0}, \ldots, \bar{d}_{p}\right)$. Then

$$
\varepsilon_{n}=\sum_{i \in I_{n}} q^{2}\left(\overline{\mathbf{d}}-\mathbf{d}, t_{i}\right), \quad t_{i} \in[0,1] .
$$


Due to Lemma 4, with $\delta_{p}$ from that lemma,

$$
\|\overline{\mathbf{d}}-\mathbf{d}\|^{2} \leq \frac{1}{\delta_{p}^{2}} \max _{0 \leq m \leq p+1} \min _{\frac{m}{p+2} \leq t \leq \frac{m+1}{p+2}} q^{2}(\overline{\mathbf{d}}-\mathbf{d}, t) .
$$

Now, by $(50)$ and condition $(v)$,

$$
\max _{0 \leq m \leq p+1} \min _{\frac{m}{p+2} \leq t \leq \frac{m+1}{p+2}} q^{2}(\overline{\mathbf{d}}-\mathbf{d}, t) \leq \mathrm{const} \frac{\varepsilon_{n}}{n_{h}} .
$$

From (49), (51) and (52) we finally get

$$
\|\overline{\mathbf{d}}-\mathbf{d}\|^{2}=o_{p}(1)
$$

and $\left(\bar{\beta}_{j}-\beta_{j}\right) h_{n}^{j} \stackrel{\mathcal{P}}{\rightarrow} 0, n \rightarrow \infty, j=0, \ldots, p$. We showed (38), and Theorem 1 is proven.

Proof of Theorem 2 We divide it into two parts.

\section{Proof. 1. The case of irrational $\theta$}

Let $\theta \in(0,1) \backslash \mathbb{Q}$. Then by $(9), \hat{\beta}_{0}=Z_{\left[\theta n_{h}\right]+1, n_{h}}$. Denote $k:=\left[\theta n_{h}\right]+1$, $\left\{t_{j}, j=1, \ldots, n_{h}\right\}:=\left\{x_{j} ; j \in I_{n}\right\}$, and $F_{j}:=F_{Y \mid t_{j}}, j=1, \ldots, n_{h}$. We have

$$
Z_{j}=F_{j}^{\leftarrow}\left(U_{j}\right), j=1, \ldots, n_{h},
$$

where $U_{1}, \ldots, U_{n_{h}}$ is an i.i.d. sequence of random variables uniformly distributed on $[0,1]$, and $F_{j}^{\leftarrow}$ is the inverse distribution function. Then

$$
Z_{k, n_{h}}=F_{k^{*}}^{\leftarrow}\left(U_{k^{*}}\right), \quad k^{*}=k^{*}(w),
$$

and $U_{k^{*}}=U_{p, n_{h}}$ for a certain random $p$. By Theorem $1, Z_{k, n_{h}} \stackrel{\mathcal{P}}{\rightarrow} Q\left(\theta, x^{*}\right)$. We have $F_{Y \mid x^{*}}\left(Q\left(\theta, x^{*}\right)\right)=\theta$, and due to $(i i)^{*}, U_{p, n_{h}} \stackrel{\mathcal{P}}{\rightarrow} \theta$. Therefore $p / n_{h} \stackrel{\mathcal{P}}{\rightarrow}$ $\theta$. Now, a simple modification of the result (3.8) from Beirlant et al. (2004b) implies that

$$
\sqrt{n_{h}}\left(U_{p, n_{h}}-\theta\right) \stackrel{\mathcal{D}}{\rightarrow} N(0, \theta(1-\theta))
$$

From a Taylor series expansion and condition $(i i)^{*}$ we have

$$
Z_{k, n_{h}}=F_{k^{*}}^{\leftarrow}\left(U_{p, n_{h}}\right)=F_{k^{*}}^{\leftarrow}(\theta)+\frac{1}{f_{k^{*}}\left(Q\left(t_{k^{*}}\right)\right)}\left(U_{p, n_{h}}-\theta\right)+\left(U_{p, n_{h}}-\theta\right) o_{p}(1) .
$$

Here $f_{S}$ is the density of the distribution function $F_{S}$. Now,

$$
\sqrt{n_{h}}\left(Z_{k, n_{h}}-Q\left(t_{k^{*}}\right)\right)=\sqrt{n_{h}}\left(U_{p, n_{h}}-\theta\right)\left(\frac{1}{f_{k^{*}}\left(Q\left(t_{k^{*}}\right)\right)}+o_{p}(1)\right),
$$


and due to $(53)$ and since $f_{k^{*}}\left(Q\left(t_{k^{*}}\right)\right) \stackrel{\mathcal{P}}{\rightarrow} f_{Y \mid x^{*}}\left(Q\left(x^{*}\right)\right)$ we have

$$
\sqrt{n_{h}}\left(Z_{k, n_{h}}-Q\left(t_{k^{*}}\right)\right) \stackrel{\mathcal{D}}{\rightarrow} N\left(0, \sigma_{\infty}^{2}\right)
$$

with

$$
\sigma_{\infty}^{2}:=\frac{\theta(1-\theta)}{f_{Y \mid x^{*}}^{2}\left(Q\left(x^{*}\right)\right.}
$$

From $(i v)^{*}$ and (2) (for $p=0$ ) we have

$$
\sqrt{n_{h}}\left|Q\left(t_{k^{*}}\right)-Q\left(x^{*}\right)\right| \leq \text { const } \sqrt{n_{h} h_{n}^{2 \gamma}} \rightarrow 0,
$$

as $n \rightarrow \infty$. Then by Slutsky's lemma, $\sqrt{n_{h}}\left(\hat{\beta}_{0}-Q\left(x^{*}\right)\right) \stackrel{\mathcal{P}}{\rightarrow} N\left(0, \sigma_{\infty}^{2}\right)$.

\section{The case of rational $\theta$}

Let $0<\theta_{1}<\theta<\theta_{2}<1$, and $\theta_{1}, \theta_{2}$ irrational. For large enough $n_{h}$,

$$
\hat{\lambda}_{1}:=Z_{\left[\theta_{1} n_{h}\right]+1, n_{h}} \leq Z_{\left[\theta n_{h}\right], n_{h}} \leq Z_{\left[\theta_{2} n_{h}\right]+1, n_{h}}=: \hat{\lambda}_{2} .
$$

But $\hat{\lambda}_{1}$ and $\hat{\lambda}_{2}$ are the estimators of the same kind as $\hat{\beta}_{0}$, for different quantiles $\theta_{1}$ and $\theta_{2}$. If $\theta_{1}$ and $\theta_{2}$ are close enough to $\theta$, then due to $(i i)^{*}$, the conditions of Theorem 1 hold true for these quantile estimators as well. Therefore, by Theorem 1 ,

$$
\hat{\lambda}_{1} \stackrel{\mathcal{P}}{\rightarrow} Q\left(\theta_{1}, x^{*}\right) \text { and } \hat{\lambda}_{2} \stackrel{\mathcal{P}}{\rightarrow} Q\left(\theta_{2}, x^{*}\right) .
$$

But due to $(i i)^{*}$,

$$
Q\left(\theta_{i}, x^{*}\right) \rightarrow Q\left(\theta, x^{*}\right), \text { as } \theta_{i} \rightarrow \theta, i=1,2 .
$$

Relations (54) to (56) imply that

$$
Z_{\left[\theta n_{h}\right], n_{h}} \stackrel{\mathcal{P}}{\rightarrow} Q\left(\theta, x^{*}\right), \text { as } n \rightarrow \infty .
$$

Then similarly to part 1 of the proof,

$$
\sqrt{n_{h}}\left(Z_{k, n_{h}}-Q\left(\theta, x^{*}\right)\right) \stackrel{\mathcal{D}}{\rightarrow} N\left(0, \sigma_{\infty}^{2}\right),
$$

for $k=\left[\theta n_{h}\right]$. But in the part 1 of the proof, we actually showed (57) for $k=\left[\theta n_{h}\right]+1$ and any $\theta$. Next (9) implies that

$$
Z_{\left[\theta n_{h}\right], n_{h}} \leq \hat{\beta}_{0} \leq Z_{\left[\theta n_{h}\right]+1, n_{h}} .
$$

Finally (58) and convergence (57) for $k=\left[\theta n_{h}\right]$ and $k=\left[\theta n_{h}\right]+1$ imply that $\sqrt{n_{h}}\left(\hat{\beta}_{0}-Q\left(\theta, x^{*}\right)\right) \stackrel{\mathcal{P}}{\rightarrow} N\left(0, \sigma_{\infty}^{2}\right)$. 


\section{Proof of Theorem 3}

Proof. Let

$$
\left\{W_{j}, j=1, \ldots, n_{h}\right\}:=\left\{Y_{i}-\sum_{j=1}^{p} \tilde{\beta}_{j}\left(x_{i}-x^{*}\right)^{j}, i \in I_{n}\right\} .
$$

Considering the right and left partial derivatives $\frac{\partial H_{n}\left(\tilde{\beta}_{0}, \tilde{\boldsymbol{\beta}}_{1}\right)}{\partial b_{0}}$, similarly to $(9)$ we obtain that

$$
\tilde{\beta}_{0}=\left\{\begin{array}{l}
W_{\left[\theta n_{h}\right]+1, n_{h}}, \text { if } \theta n_{h} \notin \mathbb{N} \\
\text { any point from }\left[W_{\theta n_{h}, n_{h}}, W_{\theta n_{h}+1, n_{h}}\right], \text { if } \theta n_{h} \in \mathbb{N}
\end{array}\right.
$$

Now, due to the structure of the parameter set $\tilde{\Theta}_{n}$, for $i \in I_{n}$ we have

$$
\left|\sum_{j=1}^{p} \tilde{\beta}_{j}\left(x_{i}-x^{*}\right)^{j}\right| \leq\left(L_{1}+\cdots+L_{p}\right) h_{n}^{\tau}=: \delta_{n} .
$$

Now, (59) and (60) imply that

$$
Z_{\left[\theta n_{h}\right], n_{h}}-\delta_{n} \leq \tilde{\beta}_{0} \leq Z_{\left[\theta n_{h}\right]+1, n_{h}}+\delta_{n} .
$$

From the proof of Theorem 2 it follows that both for $k=\left[\theta n_{h}\right]$ and $k=$ $\left[\theta n_{h}\right]+1$,

$$
\sqrt{n_{h}}\left(Z_{k, n_{h}}-Q\left(\theta, x^{*}\right)\right) \stackrel{\mathcal{D}}{\rightarrow} N\left(0, \sigma_{\infty}^{2}\right) .
$$

Moreover, by condition (vii), $\sqrt{n_{h}} \delta_{n} \rightarrow 0$, as $n \rightarrow \infty$. Then by Slutsky's lemma, both for $k=\left[\theta n_{h}\right]$ and $k=\left[\theta n_{h}\right]+1$,

$$
\sqrt{n_{h}}\left(Z_{k, n_{h}} \pm \delta_{n}-Q\left(\theta, x^{*}\right)\right) \stackrel{\mathcal{D}}{\rightarrow} N\left(0, \sigma_{\infty}^{2}\right) .
$$

Relations (61) and (62) imply the statement of Theorem 3.

\section{Conclusion}

We gave the conditions for the consistency of the estimator of the conditional quantile based on local polynomial approximations to the true conditional quantile function. We showed the asymptotic normality as well, but for the sake of this we modified the parameter set in the case $p \geq 1$.

It will be interesting to expand the results for the estimator equal $\arg \min _{\mathbf{b} \in \mathbb{R}^{p+1}} H_{n}(\mathbf{b})$, where the unconstrained optimization is performed. 


\section{Acknowledgements}

Yuri Goegebeur was supported by the Fund for Scientific Research - Flanders (F.W.O. Vlaanderen). Alexander Kukush was supported by senior postdoctoral fellowship from the Academic Authorities of the Katholieke Universiteit Leuven, Belgium.

\section{References}

[1] Beirlant, J., Goegebeur, Y., Segers, J. and Teugels, J., 2004a. Statistics of extremes - theory and applications. Wiley Series in Probability and Statistics. Wiley \& Sons, Chichester.

[2] Beirlant, J., de Wet, T. and Goegebeur, Y., 2004b. Nonparametric estimation of extreme conditional quantiles. Journal of Statistical Computation and Simulation, 74, 567-580.

[3] Fan, J., Hu, T.-C. and Truong, Y.K., 1994. Robust non-parametric function estimation. Scandinavian Journal of Statistics, 21, 433-446.

[4] Koenker, R. and Bassett, G., 1978. Regression quantiles. Econometrica, 46, 33-50.

[5] Ibragimov, I.A. and Hasminskii, R.Z., 1981. Statistical estimation: asymptotic theory. Springer, Berlin.

[6] Rosenthal, H.P., 1970. On the subspaces of $L^{p}(p>2)$ spanned by sequences of independent random variables. Isr. J. Math., 8, 267-303. 
